Typeset with jpsj2.cls $<$ ver.1.1 $>$

\title{
Riddled-like Basin in Two-Dimensional Map for Bouncing Motion of an Inelastic Particle on a Vibrating Board
}

\author{
Shohei FuKAnO*, Yumino HAYASE ${ }^{\dagger}$, and Hiizu NAKANISHI ${ }^{\ddagger}$ \\ Department of Physics, Kyushu University 33, Fukuoka 812-8581
}

(Received March 22, 2002 )

\begin{abstract}
Motivated by bouncing motion of an inelastic particle on a vibrating board, a simple two-dimensional map is constructed and its behavior is studied numerically. In addition to the typical route to chaos through a periodic doubling bifurcation, we found peculiar behavior in the parameter region where two stable periodic attractors coexist. A typical orbit in the region goes through chaotic motion for an extended transient period before it converges into one of the two periodic attractors. The basin structure in this parameter region is almost riddling and the fractal dimension of the basin boundary is close to two, i.e., the dimension of the phase space.
\end{abstract}

KEYWORDS: transient chaos, boundary crisis, riddled basin, bouncing inelastic particle, granular material

Particle motion accelerated by a vibrating boundary has been studied for some time as an example of simple physical systems that show chaotic behavior ${ }^{1,2)}$ since Fermi proposed a novel mechanism of acceleration of a charged particle through an oscillating field (Fermi acceleration). ${ }^{3)}$ A variant of the system is a bouncing inelastic particle on a vibrating board, and this has also been studied, particularly in connection with granular systems. It has been demonstrated experimentally ${ }^{4-6)}$ and theoretically ${ }^{7-10)}$ that even a single particle bouncing on a board shows a variety of behaviors such as mode-locked periodic motion, periodic doubling, chaotic behavior and quasi-periodic motion.

In this letter, based on the observation of dynamics on a single inelastic particle motion under gravity driven by a vibrating board, we construct a simple two-dimensional map, and demonstrate numerically that the map shows peculiar behavior, i.e., a very long transient chaos and a riddled-like basin structure in the parameter region where two periodic attractors coexist.

The original system we look at in constructing our map is an inelastic particle under the gravity; the particle bounces vertically on a vibrating board with a restitution constant $r$. Then, the particle velocity just before the $n$ 'th bounce $u_{n}$ and the velocity just after the bounce $v_{n}$ are related by

$$
-\frac{v_{n}-V\left(t_{n}\right)}{u_{n}-V\left(t_{n}\right)}=r
$$

where $t_{n}$ is the time at the $n$ 'th bounce and $V(t)$ is the velocity of the board at time $t$ (Fig.1).

\footnotetext{
* E-mail: fukano@stat.phys.kyushu-u.ac.jp

† E-mail: yumino@stat.phys.kyushu-u.ac.jp

‡ E-mail: naka4scp@mbox.nc.kyushu-u.ac.jp
} 
If the motion of the board $V(t)$ is given, we can determine, in principle, the time $t_{n+1}$ and the particle velocity $u_{n+1}$ of the $n+1$ 'th bounce from $t_{n}$ and $v_{n}$, and we should be able to obtain a map that describes the particle motion by using this model. It is, however, impossible in general to express the map in an explicit form ${ }^{10)}$ because the board is moving. Here, we employ the following simplification that allows us to construct the explicit map by which we can calculate $\left(v_{n+1}, t_{n+1}\right)$ from $\left(v_{n}, t_{n}\right)$.

First, we ignore the difference in the position of the board at each bounce; then we have

$$
u_{n+1}=-v_{n}, \quad t_{n+1}=t_{n}+\frac{2}{g} v_{n},
$$

where $g$ denotes the gravitational acceleration. This simplification should be justified when the amplitude of the vibration is small compared with the bouncing height (high bounce approximation). Under this simplification, the velocity just after the bounce must be positive such that $t_{n+1}>t_{n}$. To ensure this, we must assume that the vibrating board always moves upward, i.e., $V(t) \geq 0$. This appears to contradict the first simplification, but we may consider that the board shifts downward discontinuously at each period. In the present work, we employ

$$
V(t)=A(\sin \omega t+1)
$$

then we have

$$
\left\{\begin{aligned}
v_{n+1} & =r v_{n}+(r+1) A\left(\sin \omega t_{n+1}+1\right) \\
t_{n+1} & =t_{n}+\frac{2}{g} v_{n} .
\end{aligned}\right.
$$

After rescaling the variables by the transformation,

$$
v_{n} \rightarrow A v_{n}, \quad t_{n} \rightarrow \frac{A}{g} t_{n}, \quad \omega \rightarrow \frac{g}{A} \omega
$$

we have the two-dimensional map for $v_{n}$ and $t_{n}$

$$
\left\{\begin{array}{l}
v_{n+1}=r v_{n}+(1+r)\left(\sin \omega t_{n+1}+1\right) \\
t_{n+1}=t_{n}+2 v_{n}
\end{array}\right.
$$

with the two parameters $r$ and $\omega$. This was found to be equivalent to the one studied by Everson, ${ }^{7)}$ but with different parameterization.

This map shows a typical route to chaos via periodic doubling from the mode-locking bounce with the same period with the external vibration as we change the parameter $\omega$ from 0.9 to 1.8 by fixing $r=0.39$ (Fig.2a). This periodic doubling and chaotic behavior have previously been reported for the inelastic bounce system in numerical ${ }^{7,8)}$ and also experimental ${ }^{4-6)}$ studies. The strange attractor for the chaotic motion at $\omega=1.54$ and $r=0.39$ is shown in Fig.2b, which indicates that the attractor has a fractal structure with a Housdorff dimension larger than 1 in the two-dimensional phase space. 
Figure 2(b) also shows a period one attractor and its basin; this period one motion is locked to half the vibration frequency. Upon changing the parameters further, this basin moves towards the chaos attractor and causes sudden destruction of the chaos through a boundary crisis when the basin touches the chaos attractor, then the chaos disappears as a true attractor, but its vestige remains as a transient one.

After the destruction of the chaos, the period one attractor bifurcates into period two, while a new period ten attractor is stabilized out of the transient chaos and coexists with this period two attractor. In Fig.3, both attractors are shown in the phase space as well as the "temporal attractor" of the transient chaos which remains after the crisis. As is seen in this figure, the period ten attractor is embedded in the transient attractor of the chaos. In this parameter region, where the period two and the period ten orbits coexist, the system shows peculiar behavior, as we describe below.

First, the system goes through an extremely long transient period before the trajectory converges on one of the two periodic orbits, if the initial state is chosen outside the direct basin of the period two attractor. The length of the transient easily exceeds one thousand iterations. The reason for this unusually long transient is that the region of direct attraction around the period ten attractor is extremely small.

Second, the basin structures of these attractor are scattered and mixed each other almost completely (Fig.4). To characterize the structure of the basins, the uncertainty exponent, or final state sensitivity, $\alpha$ is introduced as the exponent for the probability $f(\epsilon)$ that two points separated from each other by the distance $\epsilon$ in the phase space are in different basins:

$$
f(\epsilon) \sim \epsilon^{\alpha} .
$$

The small value of $\alpha$ implies that the two basins are mixed each other rather wildly. The probability $f(\epsilon)$ is plotted in Fig.5 for $\omega=1.5852$ and $r=0.39$, and the estimated value of $\alpha$ is $6.2 \times 10^{-4}$. This means the fractal dimension $d_{f}$ of the basin boundary is very close to 2 since the uncertainty exponent $\alpha$ is related to $d_{f}$ by

$$
d_{f}=d-\alpha
$$

where $d$ is the spatial dimension of the phase space. ${ }^{2)}$

The typical appearance of the basin structure of the period ten attractor in Fig.4 strongly resembles the riddled basin, i.e., a basin that is riddled with holes. ${ }^{11,12)}$ Mathematically, the riddled basin is characterized by the following two features: (i) it has nonzero measure, (ii) any open neighbor of a point in the basin contains a point that does not belong to the basin. Such a basin has been found for a chaos attractor that is confined within a subspace of the whole phase space due to the symmetry of the system. According to this definition, a riddled basin is not an open 
set, therefore the present case cannot be a riddled basin; the basins for both the period two and the period ten attractors are open because the attractors have a finite neighbor around them that is contained within the basin, therefore whole basins can be constructed by the union of infinite pre-images of these neighbors.

On the other hand, the basin shown in Fig.4 cannot be distinguished from a riddled basin by almost any direct measure that can be calculated from the geometry of the basin. Any numerical evidence that the present basin is an open set is wiped away by the fragmentation of the pre-images during the extremely long lifetime of the transient chaos. This situation, however, does not seem to be unusual for the periodic window in the chaos; in such cases, the unstable periodic orbit embedded in the chaos attractor is stabilized extremely weakly, as in the present case.

In summary, we have examined a simple two-dimensional map that describes an inelastic ball bouncing on a vibrated board, and found that the system shows a riddled-like basin structure. The basin should not be a riddled basin based on the mathematical definition, but its uncertainty exponent is very close to 2 and it almost cannot be distinguished from a riddled basin. This is due to the very long lifetime of the transient chaos. This situation, however, seems to be generic when the unstable periodic orbit in the chaos attractor is stabilized. Note that the mechanism does not require any symmetry in the system in contrast with the case of the riddled basin.

The authors are grateful to Professor Kaneko for his useful comments.

1) M.A. Lieberman and A.J. Lichtenberg: Phys. Rev. A 5 (1972) 1852.

2) A.J. Lichtenberg and M.A. Lieberman: "Regular and Chaotic Dynamics", (Springer-Verlag, New York, 1992).

3) E. Fermi: Phys. Rev. 75 (1949) 1169.

4) P. Pierański: J. Physique 44 (1983) 573.

5) P. Pierański, Z. Kowalik and M. Franaszek: J. Physique 46 (1985) 681.

6) Z. Kowalik, M. Franaszek and P. Pierański: Phys. Rev. A 37 (1988) 4016.

7) R.M. Everson: Physica 19D (1986) 355.

8) G.A. Luna-Acosta: Phys. Rev. A 42 (1990) 7155.

9) A. Mehta and J.M. Luck: Phys. Rev. Lett. 65 (1990) 393.

10) J.M. Luck and A. Mehta: Phys. Rev. E 48 (1993) 3988.

11) A.S. Pikovsky and P. Grassberger: J. Phys. A 24 (1991) 4587.

12) E. Ott, J.C. Alexander, I. Kan, J.C. Sommerer and J.A. Yorke: Physica D 76 (1994) 384. 
Shohei Fukano,Yumino HaYase, and Hiizu NaKanishi
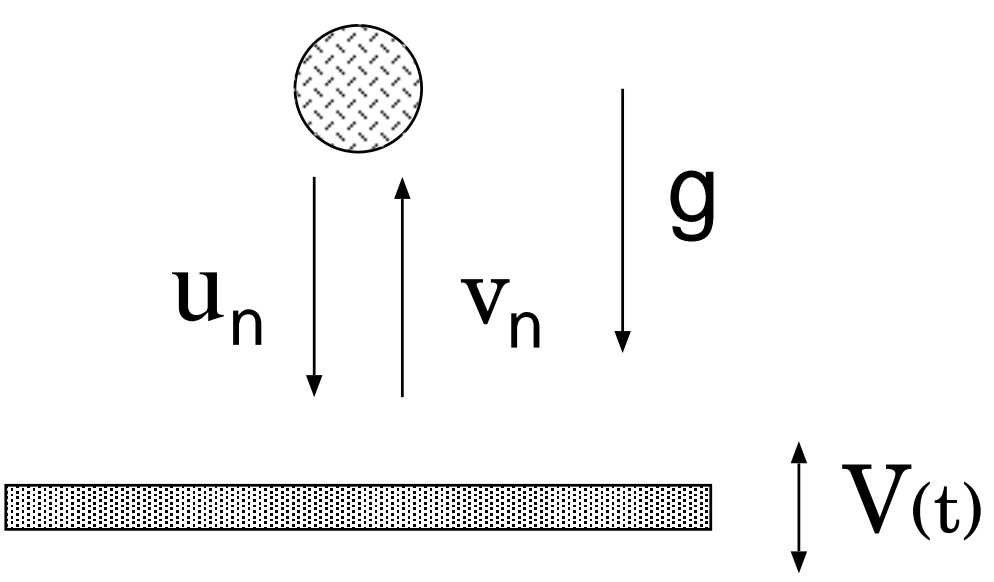

Fig. 1. An inelastic particle bouncing on a vibrating board. 


\section{Shohei Fukano,Yumino Hayase, and Hiizu NAKanishi}
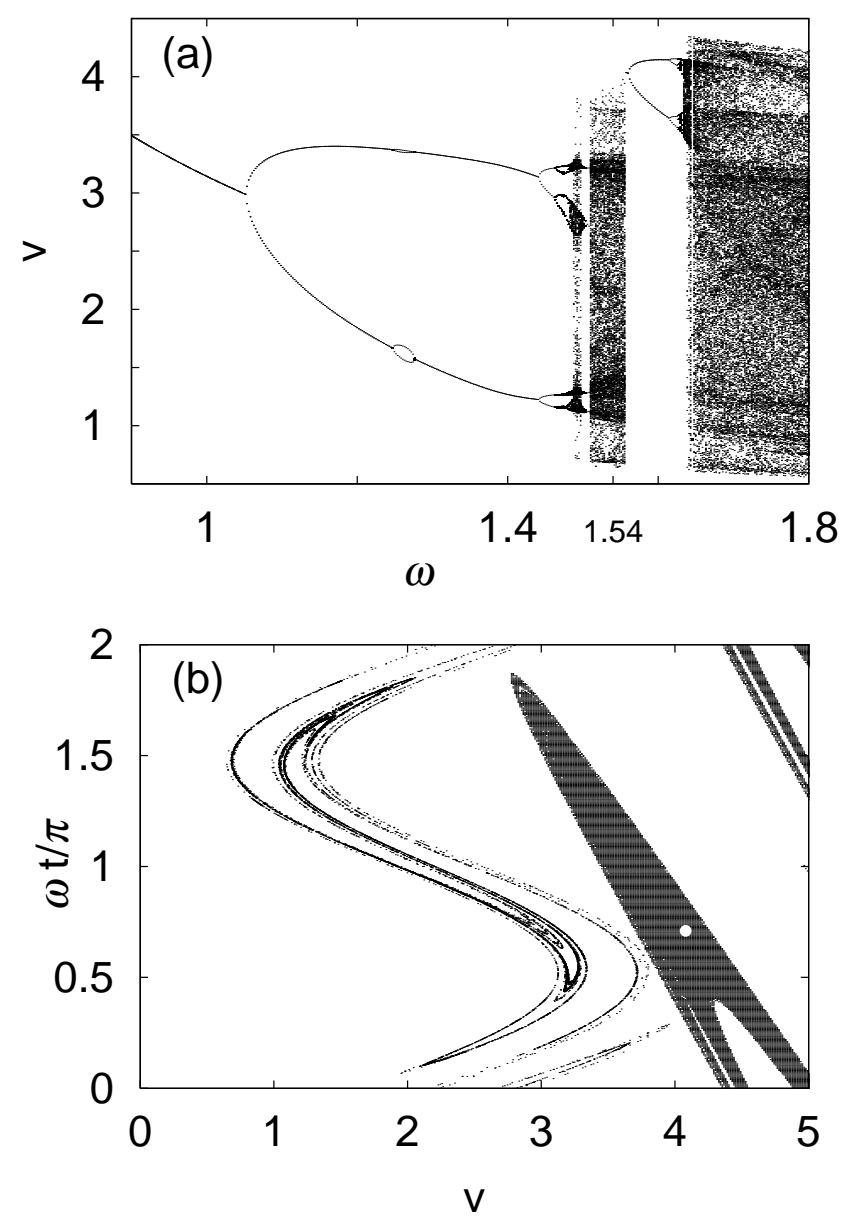

Fig. 2. (a) Bifurcation diagram upon changing $\omega$ with $r=0.39$. (b) The chaos attractor (dots) and the coexisting period one attractor (open circle) with its basin (filled area) for $\omega=1.54$ and $r=0.39$. 


\section{Shohei Fukano,Yumino Hayase, and Hiizu NAKANishi}

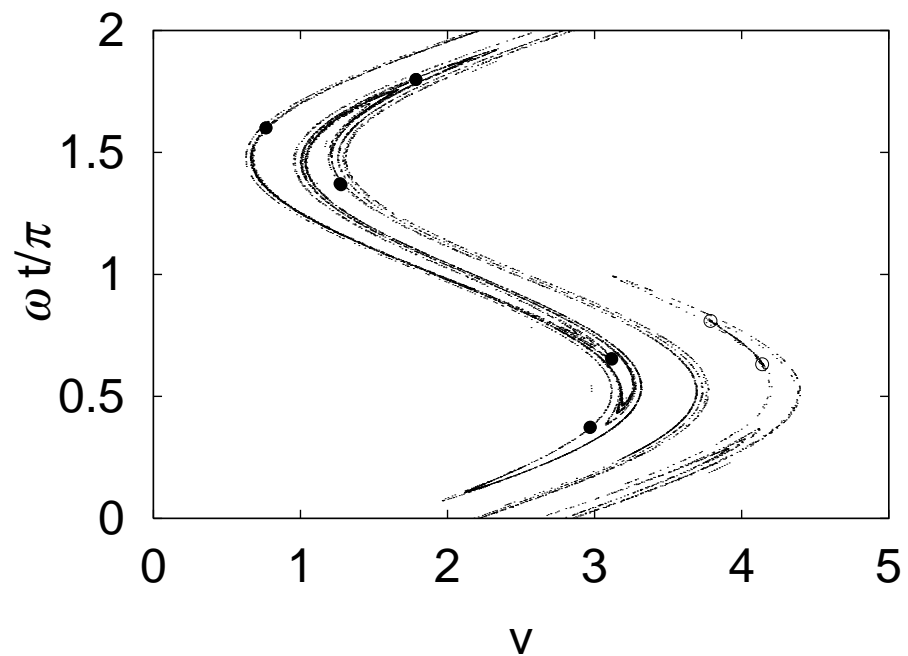

Fig. 3. The period ten attractor (solid circles) and the period one attractor (open circles) with the temporal attractor of the transient chaos (dots) for $\omega=1.5852$ and $r=0.39$ The orbit of the period ten attracter consists of five sets of two points, but the two points within each set cannot be distinguished in the figure because these two points are very close to each other. 


\section{Shohei Fukano,Yumino Hayase, and Hiizu NaKanishi}

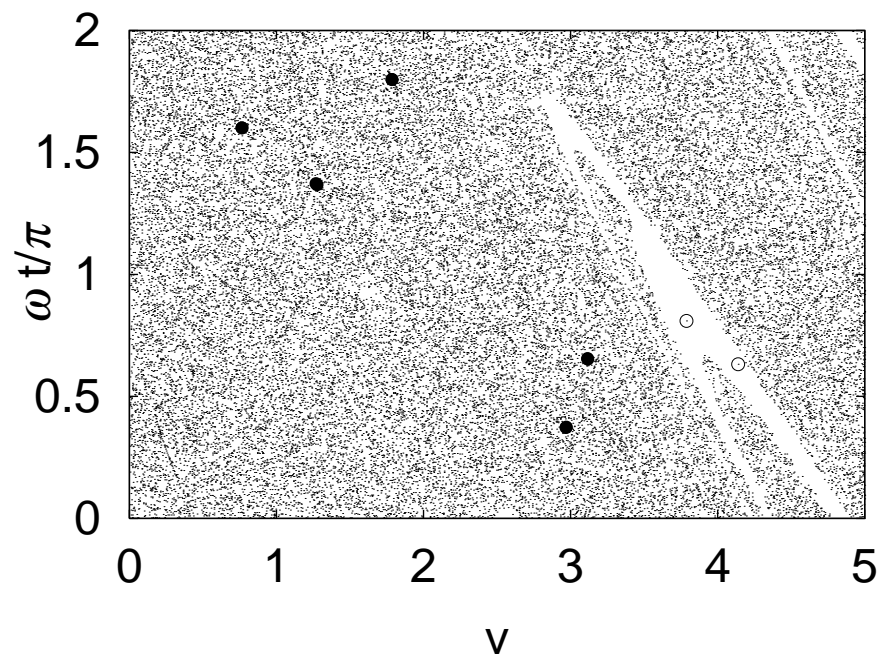

Fig. 4. The basin of the period ten attractor (dots) for $\omega=1.5852$ and $r=0.39$. The attractors of the period ten (solid circles) and the period two (open circles) orbits are also indicated. 


\section{Shohei Fukano,Yumino Hayase, and Hiizu NAKanishi}

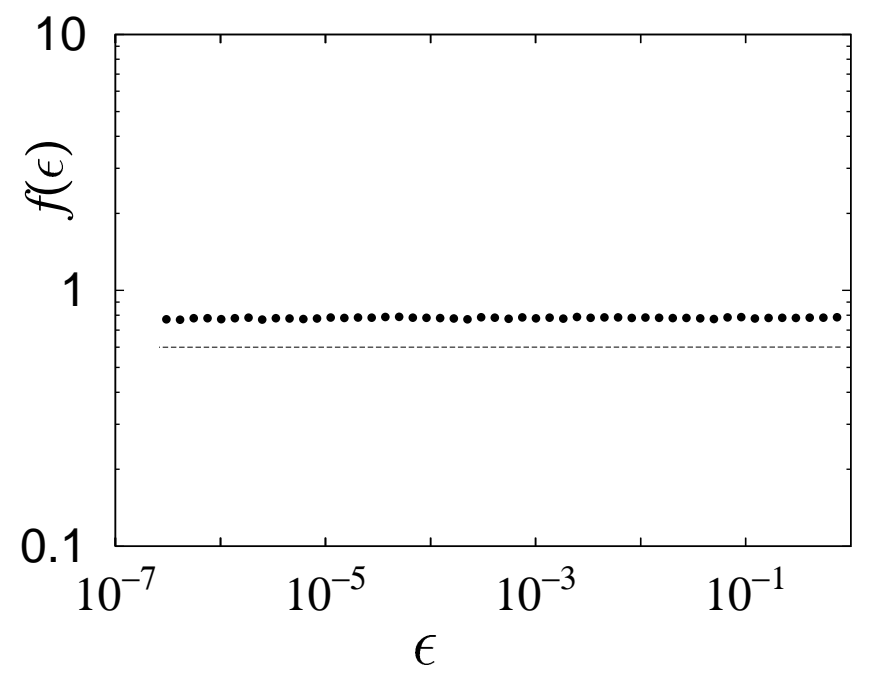

Fig. 5. The logarithmic plot of the probability $f(\epsilon)$ that two points separated by $\epsilon$ belong to different basins, for $\omega=1.5852$ and $r=0.39$. The dashed line is the line with the uncertainty exponent $\alpha=6.2 \times 10^{-4}$. 